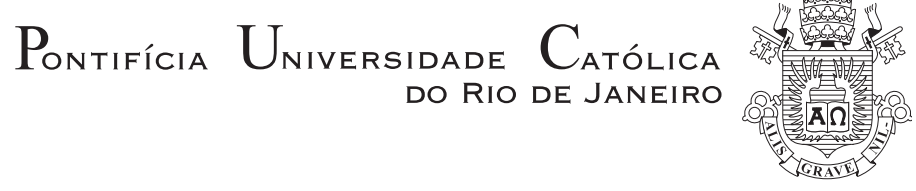

Sérgio Ricardo Kokay Morikawa

\title{
Acelerômetro Triaxial a Redes de Bragg
}

Tese de Doutorado

Tese apresentada como requisito parcial para obtenção do título de Doutor pelo Programa de Pós-graduação em Engenharia Mecânica da PUC-Rio.

Orientador: Arthur Martins Barbosa Braga Co-Orientador: Luiz Carlos Guedes Valente 


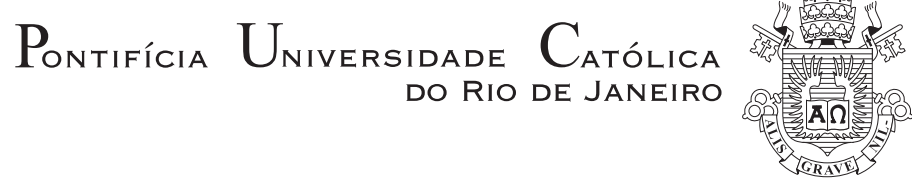

Sérgio Ricardo Kokay Morikawa

\title{
Acelerômetro Triaxial a Redes de Bragg
}

Tese apresentada como requisito parcial para obtenção do grau de Doutor pelo Programa de Pós-graduação em Engenharia Mecânica do Departamento de Engenharia Mecânica do Centro Técnico Científico da PUC-Rio. Aprovada pela Comissão Examinadora abaixo assinada.

\author{
Arthur Martins Barbosa Braga \\ Orientador \\ Departamento de Engenharia Mecânica - PUC-Rio \\ Luiz Carlos Guedes Valente \\ Co-Orientador \\ Gavea Sensors \\ Hans Ingo Weber \\ Pontifícia Universidade Católica do Rio de Janeiro \\ José Luiz de França Freire \\ Pontifícia Universidade Católica do Rio de Janeiro
}

Moysés Zindeluk

COPPE/UFRJ

Antonio Lopes Gama

Universidade Federal Fluminense

Luiz Cláudio de Marco Meniconi

CENPES/PETROBRAS

José Eugenio Leal

Coordenador Setorial do Centro Técnico Científico -

PUC-Rio

Rio de Janeiro, 31 de Maio de 2004 
Todos os direitos reservados. É proibida a reprodução total ou parcial do trabalho sem autorização da universidade, do autor e do orientador.

\section{Sérgio Ricardo Kokay Morikawa}

Graduou-se em Engenharia Mecânica na Universidade Federal do Pará em 1998. Recebeu o título de Mestre em Engenharia Mecânica pela PUC-Rio em 2000. Tem como áreas de interesse a mecânica dos sólidos, vibrações mecânicas e a óptica aplicada.

Ficha Catalográfica

Morikawa, Sérgio Ricardo Kokay

Acelerômetro Triaxial a Redes de Bragg/ Sérgio Ricardo Kokay Morikawa; orientador: Arthur Martins Barbosa Braga; co-orientador: Luiz Carlos Guedes Valente. - Rio de Janeiro: PUC-Rio, Departamento de Engenharia Mecânica, 2004.

109 f.: il. ; $30 \mathrm{~cm}$

Tese (doutorado) - Pontifícia Universidade Católica do Rio de Janeiro, Departamento de Engenharia Mecânica.

Inclui referências bibliográficas.

1. Engenharia mecânica - Teses. 2. Sensores a fibra óptica. 3. Sensores a rede de Bragg. 4. Acelerômetro triaxial. 5. Vibrações mecânicas. I. Braga, Arthur Martins Barbosa. II. Valente, Luiz Carlos Guedes. III. Pontifícia Universidade Católica do Rio de Janeiro. Departamento de Engenharia Mecânica. IV. Título. 


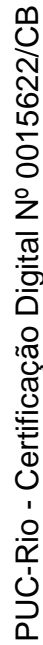

À Minha Família 


\section{Agradecimentos}

Aos meus orientadores Arthur e Guedes, pelo apoio, confiança e amizade.

A meus pais e minha irmã por seu amor incondicional.

Aos meus amigos paraenses radicados no Rio de Janeiro, pois aqui são a minha família.

Ao sensacional time do laboratório de transdutores. Adriana, Alex "O Hare", Felipe "Morpheus", "Menino" Vinícius, Rogério "Na Dele" Regazzi, Linares.

Aos membros da equipe CS. Carla "Bluetooth" Kato, Roberth "Bob" Llerena, Adriano "Pano" Pinho, Luiz "O Tocha" Nunes, Bruno "Xinxa".

A todos professores, técnicos e funcionários, que colaboraram na conclusão de meus estudos.

À Expansion - Transmissão de Energia Elétrica S. A., pela parceria e suporte financeiro.

À Agência Nacional do Petróleo pelo suporte financeiro. 


\section{Resumo}

Morikawa, Sérgio; Braga, Arthur Martins Barbosa; Valente, Luiz Carlos Guedes. Acelerômetro Triaxial a Redes de Bragg. Rio de Janeiro, 2004. 109p. Tese de Doutorado - Departamento de Engenharia Mecânica, Pontifícia Universidade Católica do Rio de Janeiro.

Desde o final da década de 80 a indústria de fibras ópticas têm passado por avanços consideráveis. Através de técnicas controladas, as fibras ópticas podem gerar sinais associados a uma vasta gama de grandezas físicas funcionando como sensores denominados de Sensores a Fibra Óptica (SFO's). Diversas técnicas podem ser empregadas para tal, e entre as existentes a baseada em redes de Bragg é a que mais tem se destacado.

O interesse por transdutores empregando esta técnica se justifica pelas vantagens proporcionadas pelo uso da luz, tais como sua capacidade de multiplexação, boa relação sinal/ruído, medições a longas distâncias, imunidade a campos eletromagnéticos, ausência de faísca, entre outras.

Neste trabalho buscou-se desenvolver um transdutor e uma técnica de medição baseada em sensores a rede de Bragg para medição de vibrações mecânicas.

Um acelerômetro óptico triaxial é projetado e construído. Diferentes modelos foram testados em busca das características de desempenho desejadas. Simulações numéricas empregando o método dos elementos finitos auxiliaram na decisão por melhores desenhos para o transdutor.

Resultados de testes experimentais e calibrações empregando um sistema de aquisição de sinais desenvolvido são mostrados. Medições de longa duração para avaliação de estabilidade do sistema e efeitos de temperatura também são apresentados.

\section{Palavras-chave}

sensores a fibra óptica, sensores a rede de Bragg, acelerômetro triaxial, vibrações mecânicas 


\section{Abstract}

Morikawa, Sérgio; Braga, Arthur Martins Barbosa; Valente, Luiz Carlos Guedes. Triaxial Bragg grating accelerometer. Rio de Janeiro, 2004. 109p. PhD. Thesis - Departamento de Engenharia Mecânica, Pontifícia Universidade Católica do Rio de Janeiro.

Since the end of the 1980s, the fiber optics industry has experienced considerable advances. Through a number of controlled techniques, fiber optics can generate signals associated with a vast array of physical measures, working as sensors denominated Optical Fiber Sensors (OFS's). Many different techniques can be employed to achieve this objective. Among these, the one based on Bragg networks has received the greatest amount of attention.

The interest in transducers employing this technique is justified by the advantages of using light, such as its multiplexing capability, good signal-to-noise ratio, possibility of long distance measurements, immunity to electromagnetic fields, and absence of sparks.

In the present work, a transducer and measurement technique based on Bragg network sensors vibration are developed, in order to measure mechanical vibrations.

A triaxial optical accelerometer is designed and built. Different models are tested in the search for the desired performance characteristics. Numerical simulations employing the finite element method help the decision making process for better transducer designs.

Results from experimental and calibration tests using a newly developed signal acquisition system are presented. Long duration measurements to evaluate system stability and temperature effects are also shown.

\section{Keywords}

optical fiber sensors, Bragg grating sensor, triaxial accelerometer, mechanical vibrations 


\section{Conteúdo}

1 Introdução $\quad 16$

\begin{tabular}{lll}
\hline 1.1 & Objetivos do trabalho & 17
\end{tabular}

$\begin{array}{lll}1.2 & \text { Os acelerômetros convencionais } & 19\end{array}$

1.3 As redes de Bragg 20

1.4 O acelerômetro óptico 24

\begin{tabular}{lll}
\hline 1.5 & Organização do trabalho & 26
\end{tabular}

2 Protótipos de acelerômetros $\quad 27$

2.1 Protótipo 128

2.2 Protótipo 2

2.3 Protótipo 3

$\begin{array}{lll}2.4 & \text { Protótipo } 4 & 33\end{array}$

2.5 Protótipo 5

3 Simulações Numéricas $\quad 38$

3.1 Características e Propriedades da Modelagem 39

3.2 Análise da Resposta em Freqüência 41

3.3 Análise de Deformações 45

4 Sistema de medição $\quad 49$

4.1 Medição em bancada 49

4.2 Sistema de leitura com duas redes de Bragg 53

4.3 Sistema de leitura com uma rede de Bragg 56

4.4 Simulação da resposta dos sensores a rede de Bragg 57

$\begin{array}{lll}5 & \text { Resultados Experimentais } & 63\end{array}$

5.1 Protótipo 1

5.2 Protótipo 2

5.3 Protótipo 3

\begin{tabular}{lll}
\hline 5.4 & Protótipo 4 & 75
\end{tabular}

$\begin{array}{lll}5.5 & \text { Protótipo } 5 & 80\end{array}$

6 Calibração e testes de estabilidade $\quad \mathbf{8 5}$

6.1 Calibração $\quad 86$

6.2 Aquisição de dados em tempo real 91

6.3 Medições de longa duração 93

$\begin{array}{lll}7 & \text { Discussões e Conclusões } & 97\end{array}$

\begin{tabular}{ll}
\hline Referências Bibliográficas & 101
\end{tabular}

A Apêndice $\quad 105$

\begin{tabular}{lll}
\hline A.1 Desenhos em CAD & 105
\end{tabular} 
$\begin{array}{ll}\text { B Apêndice } & 108\end{array}$

\begin{tabular}{ll}
\hline B.1 Programa em LabVIEW & 108
\end{tabular}

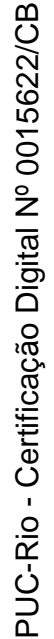




\section{Lista de Figuras}

1.1 Exemplos de possíveis aplicações. 18

1.2 Princípio das redes de Bragg. 21

1.3 Capacidade de multiplexação dos sensores a redes de Bragg. 22

1.4 Sistema de interrogação das redes de Bragg. 24

1.5 Disposição das redes em relação à massa. 25

$2.1 \quad$ Vista do protótipo $1 . \quad 28$

2.2 Montagem do protótipo $1 . \quad 29$

2.3 Vista do primeiro módulo. 30

2.4 Montagem do protótipo 2. 31

2.5 Módulo com furos passantes. 31

2.6 Montagem do protótipo 3.

2.7 Módulo com canaletas. 33

2.8 Montagem do protótipo 4. 34

2.9 Protótipo 4 encapsulado. 34

2.10 Princípio da transmissão da vibração transversal da massa sísmica. 35

2.11 Protótipo 5 - Novo módulo. 36

2.12 Encapsulamento do protótipo 5.

3.1 Modelo em elementos finitos (simplificado). $\quad 39$

3.2 Modelo em elementos finitos (completo). 40

3.3 Resultado do Protótipo 2.

3.4 Comparação entre os resultados dos protótipos 2, 3 e 4.

3.5 Análises do novo módulo para diversos valores de amortecimento $(\zeta)$. 46

3.6 Deformação calculada para o protótipo 4. 47

3.7 Deformação calculada para o protótipo 5.

4.1 Esquema empregado para as medições em laboratório. 50

4.2 Detalhe da montagem do protótipo 4 no excitador eletromagnético. 51

4.3 Espectro da fonte ASE e do LED. 52

4.4 Espectro óptico do sistema de medição com duas redes de Bragg no acelerômetro. 53

4.5 Sistema de medição com uma rede de Bragg. 56

4.6 Resultados da relação $\mathrm{V}_{1} / \mathrm{V}_{2}$ e a incerteza destes resultados [24]. 57

4.7 Caracterização dos sensores (experimental). 58

4.8 Variação da resposta AC em função da posição do filtro. $\quad 59$

$\begin{array}{ll}4.9 & \text { Simulação da relação entre a tensão AC gerada e a variação no } \\ \text { comprimento de onda. } & 59\end{array}$

4.10 Simulação do comportamento do sensor. 60

4.11 Comportamento simulado do sensor para diversos níveis de $\begin{array}{ll}\text { aceleração aplicados. } & 60\end{array}$

4.12 Resultados de simulações em amplitude e desvio de linearidade para diversas redes de Bragg utilizadas. 
4.13 Resultados de simulações para determinação da faixa dinâmica de operação.

5.1 Espectro das redes do protótipo 1.

5.2 Função de resposta em freqüência do protótipo 1.

5.3 Amortecimento do protótipo 1.

5.4 Relação sinal/ruído do protótipo 1 a $150 \mathrm{~Hz}$. 66

5.5 Linearidade da resposta do protótipo 1 a $100 \mathrm{~Hz}$ e $150 \mathrm{~Hz}$. $\quad 67$

5.6 Espectro das redes do protótipo 2.

5.7 Função de resposta em freqüência do protótipo 2 até $3200 \mathrm{~Hz}$. $\quad 68$

5.8 Anomalias na função de resposta em freqüência no módulo do protótipo 2.

5.9 Relação sinal/ruído do protótipo 2 a $200 \mathrm{~Hz}$.

5.10 Linearidade da resposta do protótipo 2 a 100,200 e $300 \mathrm{~Hz}$. $\quad 70$

5.11 Espectro das redes do protótipo 3.

5.12 Comparação da função de resposta em freqüência do protótipo 3 até $800 \mathrm{~Hz}$.

5.13 Vibração transversal do excitador eletromagnético medida durante ensaios com o protótipo 3.

5.14 Comparação da função de resposta em freqüência do protótipo 3 - Eixo transversal $x$.

5.15 Comparação da função de resposta em freqüência do protótipo 3 - Eixo transversal $y$.

5.16 Relação sinal/ruído do protótipo 3 a $200 \mathrm{~Hz}$.

5.17 Linearidade da resposta do protótipo 3. 74

5.18 Linearidade da resposta do protótipo 3 - Eixo transversal $x$. $\quad 75$

5.19 Espectro das redes do protótipo 4.

5.20 Anomalias na medição do protótipo 4. $\quad 76$

5.21 Resposta em freqüência do protótipo 4.

5.22 Espectro de potência para $50 \mathrm{~Hz}$ e $2 g$.

5.23 Espectro de potência para $100 \mathrm{~Hz}$ e $2 g$.

5.24 Linearidade da resposta do protótipo 4. $\quad 79$

5.25 Resposta transversal do protótipo 4. 80

5.26 Espectro das redes do protótipo 5.

5.27 Função de resposta em freqüência do protótipo 5 até $400 \mathrm{~Hz}$. $\quad 81$

5.28 Função de resposta em freqüência do protótipo 5 até $1600 \mathrm{~Hz}$. $\quad 82$

5.29 Alterações causadas por defeitos de montagem do módulo. $\quad 82$

5.30 Comparação entre as ressonâncias do módulo 1(a) e 2(b). $\quad 83$

5.31 Relação sinal/ruído do protótipo 5 a $200 \mathrm{~Hz}$. 83

5.32 Linearidade da resposta do protótipo 5.

6.1 Resultados experimentais do protótipo 5 comparados com a curva de calibração determinada (Módulo 1 - Sensores 1 e 2). $\quad 87$

6.2 Resultados experimentais do protótipo 5 comparados com a curva de calibração determinada (Módulo 2 - Sensores 1 e 2). $\quad 87$

6.3 Ajuste linear simulado e experimental (Módulo 1). 88

6.4 Ajuste linear simulado e experimental (Módulo 2). 88

6.5 Resultados de simulações em amplitude para diferentes valores de temperatura $\left(\lambda_{\text {filtro }}>\lambda_{\text {sensor }}\right)$. 
6.6 Simulações em amplitude de pico para diferentes valores de $\begin{array}{ll}\text { temperatura. } & 90\end{array}$

6.7 Desvio de linearidade para diferentes valores de temperatura. 90

6.8 Fluxograma de aquisição e processamento de sinais. 91

6.9 Interface gráfica da rotina em LabVIEW. 92

6.10 Teste de longa duração do acelerômetro óptico. 93

6.11 Teste de longa duração do acelerômetro óptico com interrupções. 93

6.12 Efeito da variação de temperatura nos sensores (fora de fase). $\quad 94$

6.13 Efeito da variação de temperatura nos sensores (em fase). $\quad 95$

6.14 Efeito da variação de temperatura com $\lambda_{\text {filtro }}=\lambda_{\text {sensor }}$ (durante a noite). 95

6.15 Efeito da variação de temperatura com $\lambda_{\text {filtro }}=\lambda_{\text {sensor }}$ (durante $\begin{array}{ll}\text { a madrugada). } & 96\end{array}$

A.1 Módulo do protótipo 5. 105

A.2 Massa sísmica para o protótipo 5.

A.3 Corpo para o encapsulamento do protótipo 5. 106

\begin{tabular}{lll}
\hline A.4 Tampa de vedação superior. & 107
\end{tabular}

A.5 Tampa de vedação inferior. 107

B.1 Programa principal de aquisição de sinais em LabVIEW. 108

B.2 Programa principal de aquisição de sinais em LabVIEW (continuação). 


\section{Lista de Tabelas}

3.1 Propriedades dos materiais utilizados. $\quad 41$

3.2 Constantes reais dos elementos SHELL 63 e BEAM 4. 41

3.3 Avaliação da resposta em freqüência para o novo módulo. 44

3.4 Simulação das deformações sem amortecimento $(\mu \mathrm{m} / \mathrm{m})$. $\quad 46$

6.1 Características dos sensores utilizados. 86

7.1 Características gerais de desempenho do protótipo final $\begin{array}{ll}\text { (protótipo 5). } & 99\end{array}$

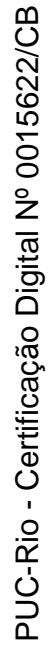




\section{Lista de Símbolos}

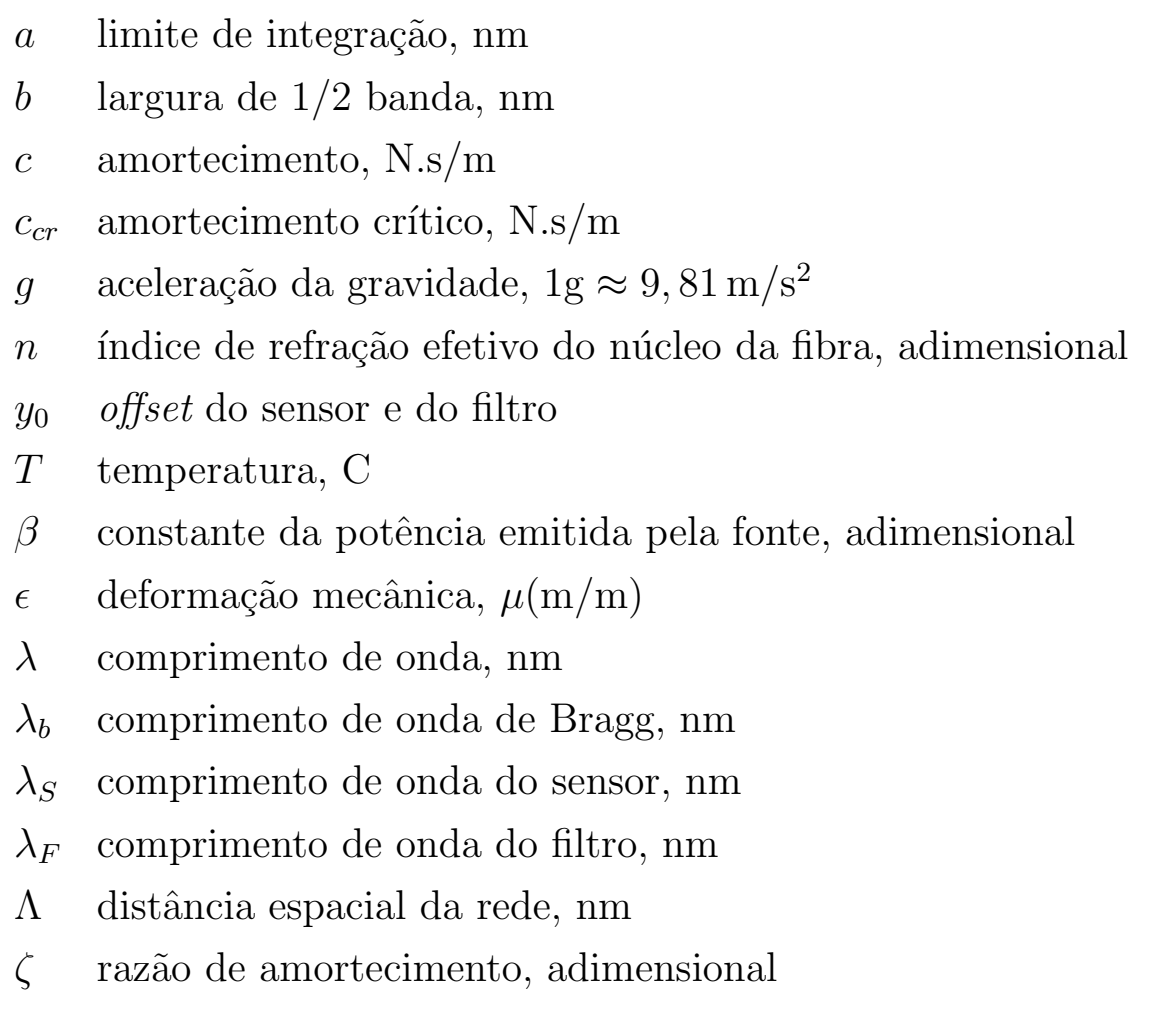


Quero que o mundo possua os frutos gloriosos de meu labor, para despertar a alma e abrir a compreensão daqueles que são privados daquela luz que, com toda certeza, não é de minha própria invenção. Caso eu caia em erro, não acredito que tenha errado de propósito. E ao falar e escrever como faço não luto pelo desejo de ser vitorioso; pois considero todo tipo de renome e conquista inimigo de Deus, vil e sem uma partícula de honra, caso não seja verdade; mas pelo amor da verdadeira sabedoria e no esforço de refletir corretamente, esgoto-me, torturo-me, atormento-me.

Giordano Bruno, O papa e o herege. 